\section{Kidney Blood Pressure Research}

Original Paper

\title{
Rhabdomyolysis-Induced Acute Kidney Injury Under Hypoxia and Deprivation of Food and Water
}

\author{
Jingwen Wang Dewen Wang Yang Li Hongyan Zuo Shaoxia Wang \\ Xinping Xu Xiaoming Guo Yabing Gao Shuiming Wang Ruiyun Peng
}

Beijing Institute of Radiation Medicine

Key Words

Rhabdomyolysis • Acute kidney injury $•$ Hypoxia $•$ Apoptosis

\begin{abstract}
Background: To investigate the renal pathophysiologyin rhabdomyolysis-induced acute kidney injury (AKI) in rats under hypoxia and deprivation of food and water (HDFW), thus broadening the knowledge about rhabdomyolysis-induced AKI in massive earthquake. Methods: Male Wistar rats weighing 200-230g were randomized into control, rhabdomyolysis (R), HDFW and rhabdomyolysis in combination with HDFW (R/HDFW) group. Experimental rhabdomyolysis rat model was established through clamping hind limb muscles, HDFW model rats were kept in $10 \%$ hypoxic chamber unavailable to food and water. At 1, 3, 5, 7, 9, 11d after treatment, serum creatinine (Scr) level, renal index, renal structural changes and cell apoptosis were analyzed. Results: After R, HDFW, R/HDFW treatment, the animals showed significantly higher Scr levels than the control group. Renal index in R and R/HDFW groups elevated remarkably compared with that in control and HDFW group. The results of histopathology, ultra-structureand apoptosis assay suggested that rhabdomyolysis caused renal tubular injury, HDFW treatment resulted in renal vascular dilation, tissue congestion and tubular cell damage. In addition, more severe renal lesion appeared in R/HDFW. Conclusions: Weconclude that the association of experimental rhabdomyolysis with HDFWresults in a different functional and histological pattern. The rhabdomyolysis-HDFW combination causes more severe renal injury.
\end{abstract}

Copyright $@ 2013$ S. Karger AG, Basel

\section{Introduction}

Acute kidney injury (AKI) has now replaced the term acute renal failure as a universal definition and staging system has been proposed to allow earlier detection and management of AKI. Clinically AKI is characterized by a rapid reduction in kidney function resulting in a failure to maintain fluid, electrolyte and acid-base homoeostasis. There have previously been many different definitions of AKI used in the literature which has made it difficult to determine the epidemiology and outcomes of AKI. Over recent years there has been 


\section{Kidney Blood Pressure Research}

Kidney Blood Press Res 2013;37:414-421

\begin{tabular}{l|l}
\hline DOI: $10.1159 / 000350154$ & (c) 2013 S. Karger AG, Basel
\end{tabular}

Published onlıne: October 10, 2013

www.karger.com/kbr

Wang, et al.: Rhabdomyolysis-induced AKI under hypoxia and deprivation of food and water

increasing recognition that relatively small rises in serum creatinine (Scr) in a variety of clinical settings are associated with worse outcomes. A variety of causes can trigger AKI including renal ischemic events, cardiac disorders and impaired systemic circulation, hepatic malfunction, sepsis, rhabdomyolysis and exposure to nephrotoxic substances.

Rhabdomyolysis-induced AKI, develops following skeletal muscle trauma, is associated with high morbidity and mortality $[1,2]$. The pathophysiology of AKI in rhabdomyolysis is likely complex and incompletely understood. Severe muscle injury (rhabdomyolysis) is accompanied by the release of myoglobin that becomes deposited in the kidney, causing renal injury. Myoglobin has been shown to bind to Tamm-Horsfall protein, especially in an acidic milieu, forming tubular casts [3]. Cast-bound myoglobin can cause oxidative damage, lead to tubular toxicity and vasoconstriction [4]. Muscle swelling, intravascular volume depletion, reduced cardiac [5] and lactic acidosis are all probably to contribute to renal injury. Renal cortical blood flow is markedly reduced in rhabdomyolysis [6]. The mechanisms involved in the pathogenesis of rhabdomyolysis are direct sarcolemmic injury (e.g., trauma) or depletion of ATP within the myocyte, leading to an unregulated increase in intracellular calcium $[7,8]$. The possibility of interactions between non-oxidant and oxidant mechanisms in rhabdomyolysis was revealed [9]. A study suggested that hypoxia occurs within the kidney and that it contributes to renal cell damage in rhabdomyolysis -induced AKI [10]. AKI associated with myoglobinuria is the most serious complication of both traumatic and non-traumatic rhabdomyolysis, and it may be life-threatening. AKI as a complication of rhabdomyolysis is quite common, representing about 7 to $10 \%$ of all cases of AKI in the United States $[11,12]$. Direct muscle trauma after natural or human-made disasters remains responsible for large-scale occurrences of rhabdomyolysis [13]. For example, after the 1988 Armenian earthquake, more than 1000 cases of rhabdomyolysis-induced myoglobinuric renal failure were reported [14]. In the 2008 Wenchuan earthquake in China, the hospitals admitted a large number of crush injury and crush syndrome victim [15].

In massive earthquake, many of the cases with rhabdomyolysis being buried under debris suffered hypoxia and limitation of food and water resource, which would lead to renal dysfunction. Obviously, rhabdomyolysis under hypoxia and deprivation of food and water (HDFW) is more clinically relevant in massive earthquake. However, to our knowledge, there have been no reports on renal changes in rhabdomyolysis in this situation. The understanding of renal changes in this process might provide new insight to clinical treatment.

In this study, in order to obtain experimental data on rhabdomyolysis-induced renal changes under the condition of HDFW in massive earthquake, renal changes in rhabdomyolysis-HDFW combination was investigated.

\section{Materials and Methods}

\section{Animals}

Male Wistar rats (200-230 g) were obtained from the center for experimental animal of our institute, kept at $22^{\circ} \mathrm{C}$ with a 12:12-h light-dark cycle and with free access to rodent chow and tap water for 3 days before the performance of the experiment. Experiments were conducted in accordance with the NIH Guide for the Care and Use of Laboratory Animals.

\section{Rhabdomyolysis}

Animal were randomly assigned into two groups: a rhabdomyolysis group and a non- rhabdomyolysis group. Rhabdomyolysis was induced by clamping the hind limb muscles under pentobarbital anaesthesia (60mg/kg IP). In brief, $41 \mathrm{~mm}$ metal clamps were used to clamp the proximal part of the hind limb(the pressure caused by the clamp was $4.5 \pm 0.3 \mathrm{Kg}$ ), the clamped limbs swelled right after clamping, on day 3 , muscle necrosis was obvious and progressed over time.

HDFW (hypoxia and deprivation of food and water)

Rats from both the rhabdomyolysis(R) and control groups were randomly divided into two groups: one group was kept in 10\% isotonic hypoxic chamber( normal humidity and temperature), without access 


\section{Kidney \\ Blood Pressure Research}

\begin{tabular}{l} 
Kidney Blood Press Res 2013;37:414-421 \\
\hline \begin{tabular}{l|l} 
DOI: 10.1159/000350154 & (c) 2013 S. Karger AG, Basel \\
Published online: October 10, 2013 & wwrger.com/kbr
\end{tabular} \\
\hline
\end{tabular}

Wang, et al.: Rhabdomyolysis-induced AKI under hypoxia and deprivation of food and water

to food and water as control/HDFW or R/HDFW, and another group was kept in normoxic environment and given normal chow and water.

\section{Kidney weight index and functional studies}

Kidneys were rapidly excised and rinsed in cold PBS. Kidney weight was measured. Kidney weight index was determined as the kidney weight to body weight ratio. Blood sampling from venae cava inferior was obtained under anaesthesia. Plasma samples were processed for the determination of creatinine and blood urea nitrogen (BUN) by automatic biochemistry analyzer (HITACHI-7170S, Japan).

\section{Histologic pathology}

The kidneys were fixed with $4 \%$ paraformaldehyde. The fixed kidney tissue was cut into 3-mm-thick blocks. The tissue blockswere embedded in paraffin and cut into $4 \mu \mathrm{m}$ slices. After being deparaffinized using xylene and ethanol dilutions and rehydration, the sections were stained with hematoxylin and eosin (H\&E), and periodic acid Schiff (PAS) staining were also performed. Histological damage of the kidney was scored semi-quantitatively using a score system ranging from 0-2 ( score 0 , no significant tubular damage, score 1 , moderate damage, score 2 , severe damage).

\section{Ultra-structural assessment}

Dissected kidney samples were fixed with $2.5 \%$ glutaraldehyde for $24 \mathrm{~h}$, cut into approximately $1 \mathrm{~mm}^{3}$ pieces. After rinsed twice for $15 \mathrm{~min}$ at a time in $0.2 \mathrm{M}$ phosphate buffer, the samples were postfixed in $1 \%$ buffered osmium tetroxide for $1 \mathrm{~h}$, then dehydrated through graded alcohols, and embedded in resin. Ultrathin sections $(50 \mathrm{~nm})$ were cut on an ultra-microtome and stained with uranyl acetate and lead citrate. Then all the sections were observed under scanning electron microscope for changes in kidney cells. Ultrathin sections were examined under the transmission electron microscope (Philips-CM100, Holland).

\section{Terminal transferase-mediated dUTP nick end-labeling (TUNEL) assay}

TUNEL staining was performed with the In Situ Cell Death Detection Kit, POD (Roche Applied Science, Mannheim, Germany). Paraffin-embedded tissue sections underwent the following protocol: heated the slides at $60^{\circ} \mathrm{C}$ followed by xylene wash and a graded series of ethanol and double distilled water washes; incubated the slides with protease $\mathrm{K}$ for $15-30$ min at $21^{\circ} \mathrm{C}-37^{\circ} \mathrm{C}$; washed the slides with $1 \%$ Triton $\mathrm{X}-100$ for $8 \mathrm{~min}$. Slides were rinsed with PBS and incubated for $60 \mathrm{~min}$ at $37^{\circ} \mathrm{C}$ with $50 \mu \mathrm{l}$ of TUNEL reaction mixture, followed by incubation for $30 \mathrm{~min}$ at $37^{\circ} \mathrm{C}$ with $50 \mu$ of converter-POD. Slides were rinsed with PBS and incubated for $10 \mathrm{~min}$ at $15^{\circ} \mathrm{C}-25^{\circ} \mathrm{C}$ with $50 \mu \mathrm{l}$ of DAB substrate solution, then rinsed with PBS again. After these treatments, the slides were analyzed under light microscopy.

\section{Statistics}

The SPSS statistical program was employed for statistical evaluation. Data are represented as means \pm SD. ANOVA with post hoc Student's $t$-test was applied for between-group comparisons of the groups. Statistical significance was set at $P<0.05$.

\section{Results}

A total of 144 animals were included into the study, randomized into 4 groups: untreated controls, R group, HDFW group, R/HDFW group ( 6 for $1,3,5,7,9$, 11 d time points, respectively). Elevated Scr and BUN reflect rhabdomyolisis-induced acute kidney injury. Apart from the animals sacrificed at indicated time points, the rats with R/HDFW died between 4.2-6.8 d after treatment, the rats from HDFW group died between 6.5-11d after treatment (Table 1).

\section{Animals}

HDFW and R/HDFW produced a significant body and kidney weight loss in the experimental process. A remarkable kidney weight index gain of $\mathrm{R}$ and R/HDWF group occurred after $5 \mathrm{~d}$ of treatment as compared with that of control group, presenting as oedema and infiltration (Figure 1A-C). 


\section{Kidney Blood Pressure Research}

Table 1. Death rate of the rats exposed to rhabdomyolysis(R), hypoxia with deprivation of food and water (HDFW) and $\mathrm{R}$ plus HDFW for different time

\begin{tabular}{lcccccc}
\hline \multirow{2}{*}{ Group } & \multicolumn{7}{c}{ Time of treatment } \\
\cline { 2 - 7 } & $1 \mathrm{~d}$ & $3 \mathrm{~d}$ & $5 \mathrm{~d}$ & $7 \mathrm{~d}$ & $9 \mathrm{~d}$ & $11 \mathrm{~d}$ \\
\hline R & 0 & 0 & 0 & 0 & 0 & 0 \\
HDFW & 0 & 0 & 0 & $9.5 \%(2 / 21)$ & $64.3 \%(9 / 14)$ & $100 \%(2 / 2)$ \\
R/HDFW & 0 & 0 & $53.3 \%(16 / 30)$ & $100 \%(9 / 9)$ & - & - \\
\hline
\end{tabular}

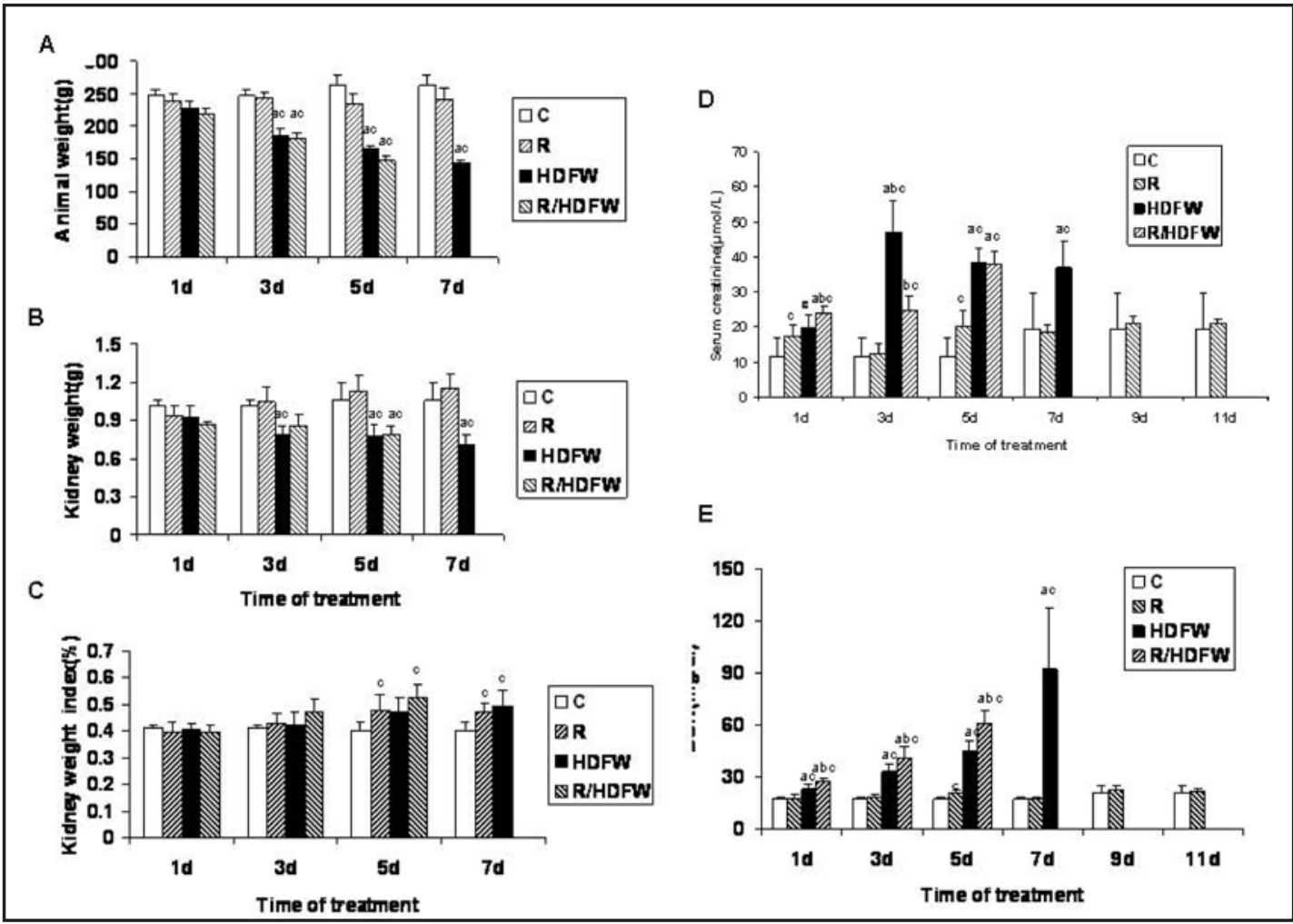

Fig. 1. Body weight (A), kidney weight (B), kidney weight index(C) , serum creatinine(D) and BUN(E) of the rats exposed to rhabdomyolysis(R), hypoxia with deprivation of food and water (HDFW) and R plus HDFW. C: control, R: rhabdomyolysis, HDFW: hypoxia with deprivation of food and water. ${ }^{a} P<0.05$ vs. $\mathrm{R},{ }^{\mathrm{b}} P<0.05$ vs. HDFW, ${ }^{c} P<0.05$ vs. control.

\section{Functional data}

In $\mathrm{R}$ group, Scr levels increased significantly on day 1 and day 5 against the control group and returned to normal on day 7, BUN value was markedly higher than control on day 5. In HDFW and R/ HDFW groups, Scr and BUN levels elevated progressively in a timedependent manner. The Scr and BUN level in R/HDFW was significantly higher than those in HDFW group, suggesting that R/HDFW produced more severe renal functional impairment (Figure 1D-E).

\section{Histopathological changes in rat kidney}

H\&E staining manifested that, R, HDFW and R/HDFW groups presented different histological patterns. With respect to the control group, the major morphologic injury in $\mathrm{R}$ group occurred on day 5, in the form of swelling, necrosis in renal tubular epithelial cells, accumulation of PAS-positive material in the tubular lumen, which were consistent with previous studies. In HDFW group, the major renal histological alterations were progressing vessel dilation (including glomerular capillary) and tissue congestion after $3 \mathrm{~d}$ of treatment, in addition, tubular damage was also seen. R/HDFW produced intense tissue injury, apart from identical alteration of vascular system to HDFW group, more severe tissue damage 


\section{Kidney Blood Pressure Research}

Wang, et al.: Rhabdomyolysis-induced AKI under hypoxia and deprivation of food and water

Table 2. Tubular damage score of the rat renal tubular cells exposed to rhabdomyolysis(R), hypoxia with deprivation of food and water (HDFW) and R plus HDFW. $0=$ no damage, $1=$ epithelium at least partially preserved, $2=$ epithelium completely damaged. ${ }^{a} P<0.05 v s . \mathrm{R},{ }^{\mathrm{b}} P<0.05 v s$. HDFW, ${ }^{\mathrm{c}} P<0.05 v$ s. control

\begin{tabular}{lccccc}
\hline \multirow{2}{*}{ Group } & \multicolumn{5}{c}{ Time of treatment } \\
\cline { 2 - 6 } & $1 \mathrm{~d}$ & $3 \mathrm{~d}$ & $5 \mathrm{~d}$ & $7 \mathrm{~d}$ & $9 \mathrm{~d}$ \\
\hline $\mathrm{R}$ & $0.0 \pm 0.0$ & $0.7 \pm 0.6^{\mathrm{c}}$ & $1.0 \pm 0.0^{\mathrm{c}}$ & $0.7 \pm 0.6^{\mathrm{c}}$ & $0.0 \pm 0.0$ \\
$\mathrm{HDFW}$ & $0.0 \pm 0.0$ & $1.0 \pm 0.0^{\mathrm{ac}}$ & $1.0 \pm 0.0^{\mathrm{c}}$ & $1.3 \pm 0.6^{\mathrm{ac}}$ & - \\
R/HDFW & $0.7 \pm 0.6^{\mathrm{c}}$ & $1.0 \pm 0.0^{\mathrm{c}}$ & $2.0 \pm 0.0^{\mathrm{abc}}$ & - & - \\
\hline
\end{tabular}

$0=$ no damage, 1 = epithelium at least partially preserved, 2 = epithelium completely damaged. a $P<0.05$ vs. $\mathrm{R}, \mathrm{b} P<0.05$ vs HDFW, $\quad{ }^{c} P<0.05$ vs. control.

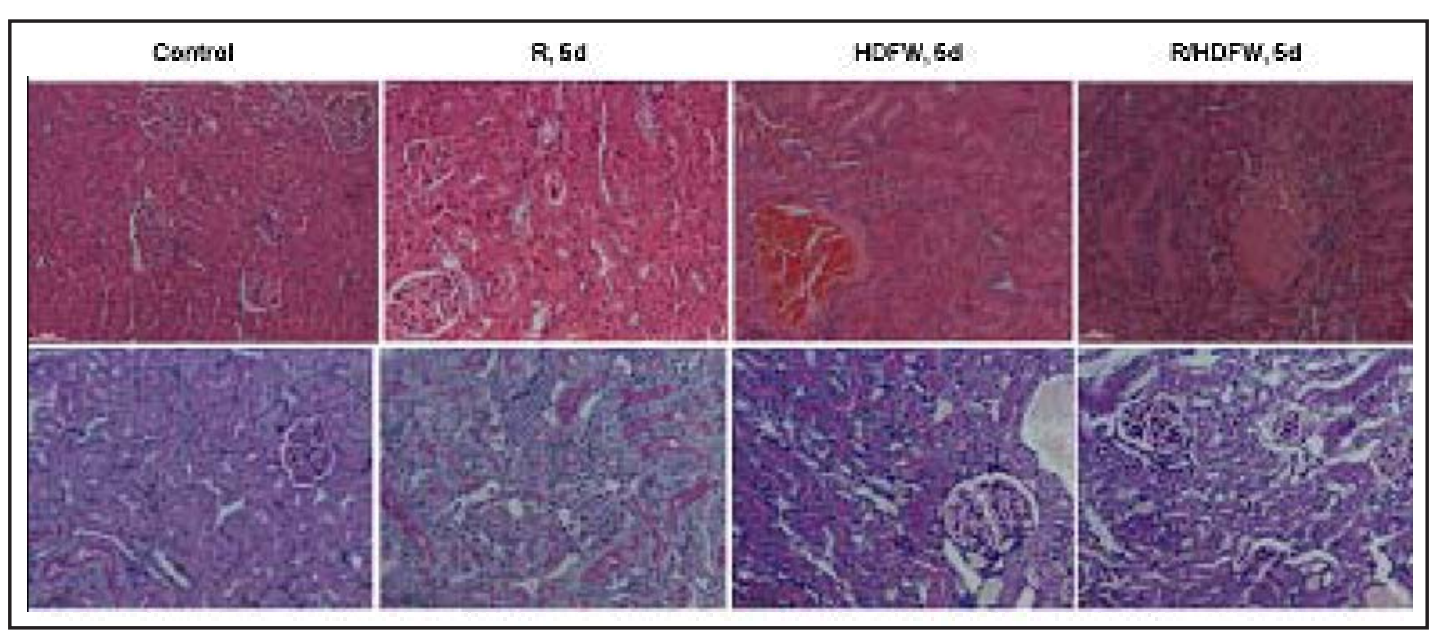

Fig. 2. Histopathological changes of the rat kidney exposed to rhabdomyolysis(R), hypoxia with deprivation of food and water (HDFW) and R plus HDFW. The kidney tissue slices were stained with hematoxylin and eosin and observed by inverted phase contrast microscope. Top panel: haematoxylin and erosin (H\&E) staining, bottom panel: periodic acid-Schiff(PAS) staining. Bar=50 $\mu \mathrm{m}$.

appeared, focal necrosis was seen in the cortex, which was peculiar pathology in this group (Figure 2, Table 2).

\section{Ultra-structural changes in rat renal tubules}

After $3 \mathrm{~d}$ of challenges, ultra-structural alterations in renal tubular cells were observed. R, HDFW and R/HDFW groups showed mitochondrial swelling with local vacuolization and chromatin accumulation in the nuclei rim in tubular epithelial cells. In HDFW and R/HDFW groups, in addition to above changes, cytoplasmatic vacuolization, nuclei condensation, capillary dilation occurred. Furthermore, renal ultra-structural changes in R/HDFW group at day 6.8 were investigated, the results disclosed that, above changes became more severe with lysosome deposit in tubular cells (Figure 3).

\section{Apoptosis in rat renal tubule}

Positive nuclear reactions in the kidneys of the R, HDFW, R/HDFW-treated and control rats were analyzed by the TUNEL staining. TUNEL-positive cells were undetectable in normal kidney, the R, HDFW, R/HDFW-treated rat kidneys showed positive staining for apoptosis. The R/HDFW treatment led to more apoptotic renal cells than either R or HDFW. In R group, the positive cells were mainly expressed in the cortex and the outer medulla, while in HDFW and R/HDFW group, the positive cells distributed in whole kidney tissue. HDFW and R/ HDFW showed a reduction in apoptosis rate from day 3 to day 5 , the reason could be that cell necrosis exceeded apoptosis (Figure 4). 


\section{Kidney Blood Pressure Research}

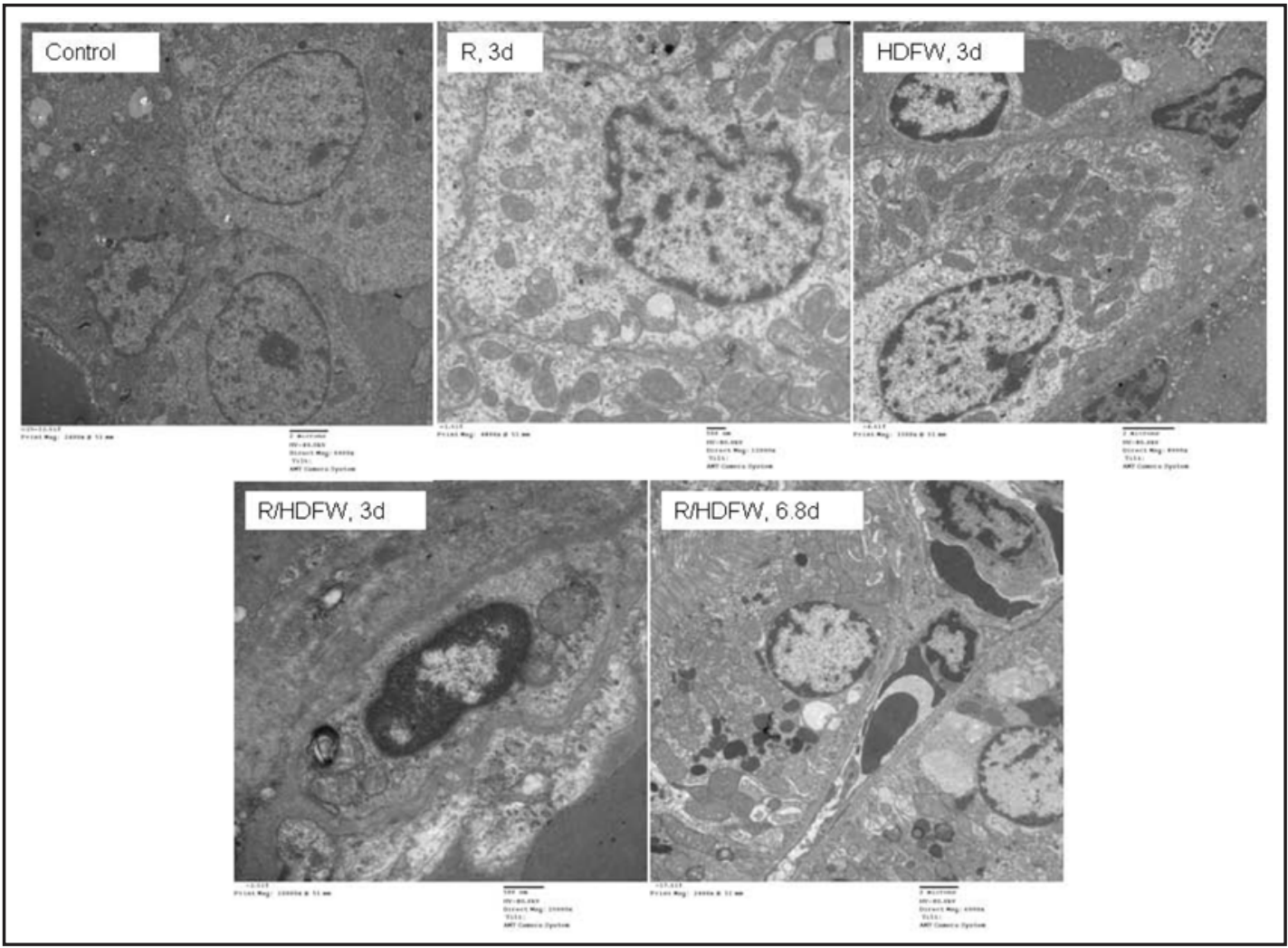

Fig. 3. Ultra-structural changes of the rat renal tubular cells exposed to rhabdomyolysis(R), hypoxia with deprivation of food and water (HDFW) and R plus HDFW.

\section{Discussion}

In this study, renal pathophysiology induced by rhabdomyolysis in combination with HDFW was firstly investigated. In massive earthquake, many cases with rhabdomyolysis under debris suffered from air loss and shortage of food and water, which made rhabdomyolysisinduced AKI more complicated. The aim of this work was to elucidate the renal pathological changes in this situation.

Rhabdomyolysis is a dissolution of skeletal muscles that produces a nonspecific clinical syndrome that causes extravasation of toxic intracellular contents from the myocytes into the circulatory system. Regardless of the initial precipitating factor, leaky skeletal muscle cells constitute the common rhabdomyolysis pathway [16-18]. Following rhabdomyolysis, inordinate amounts of myoglobin are released into the systemic circulation and its prompt discharge into the renal tubules sets the stage for the initiation of renal injury process [19, 20]. The rhabdomyolysis-induced alterations in renal histology and function in the present work are consistent with previous studies. While in our model, tubular damage was slighter than some reports, which could be helpful in exploring the effect of combined injury.

Evidence demonstrated that systemic hypoxia exposure could lead to renal changes. For example, an increase in renal vasodilatation and a decrease in glomerular filtration rate occurred in anesthetized rats subjected to moderate hypoxia [21]. In contrast, a tendency towards renal vasoconstriction in unanaesthetized rats was observed and may be explained by a greater influence of sympathetic nerve activity as a primary response to peripheral chemoreceptor stimulation, or to activation of the brainstem defense areas, or by circulating and local vasoconstrictor hormones [22]. The researchers who recorded vasodilatation consider a possible local mediator of such a differential effect is adenosine. Systemic hypoxia is known to cause the release of adenosine from many tissues including the kidney [23]. Adenosine causes dilatation of efferent arterioles by acting on A2 receptors, but may cause 


\section{Kidney \\ Blood Pressure Research}

Fig. 4. Effect of rhabdomyolysis(R), hypoxia with deprivation of food and water (HDFW) alone and combination on apoptosis in rat renal tubular epithelial cells determined by TUNEL staining. (A)Kidneys from the rats treated with R/HDFW were stain-ed using the TUNEL assay. Bar $=50 \mu \mathrm{m}$. (B) Comparison of number of apoptotic cells per 100 counted. aP $<0.05$ vs. $\mathrm{R},{ }^{\mathrm{b}} P<0.05$ vs. HDFW.

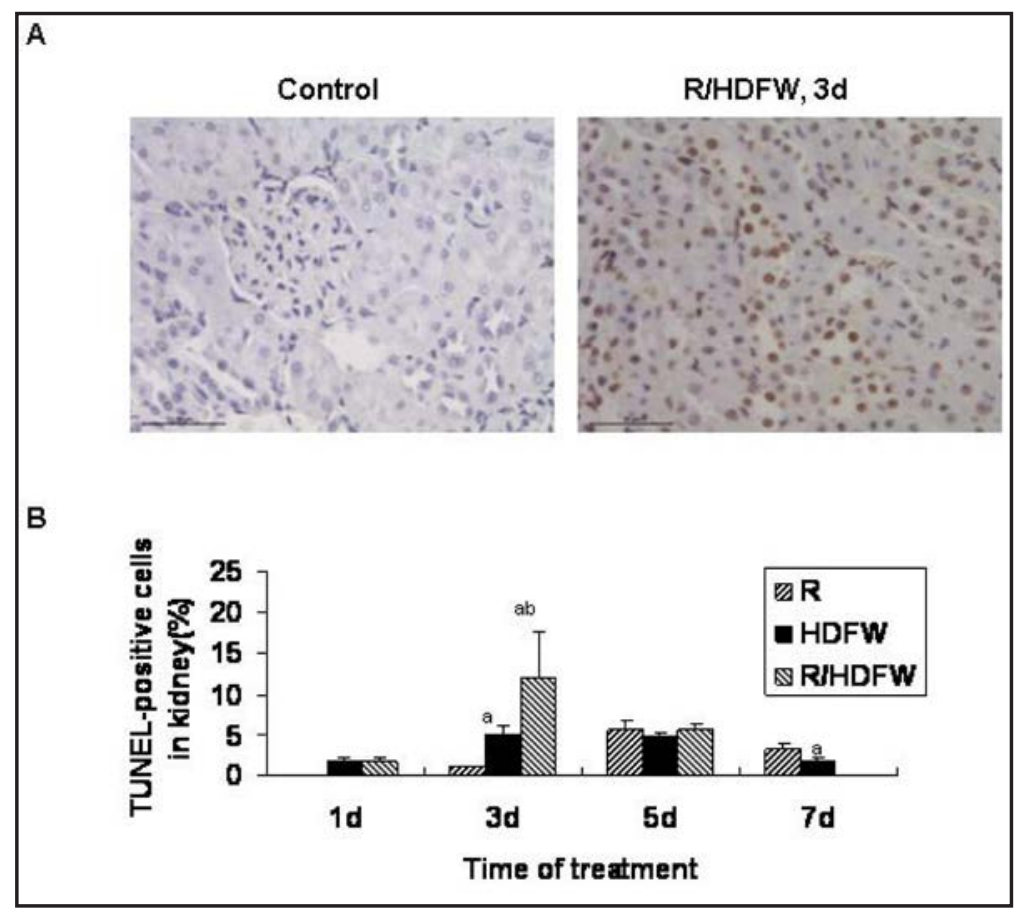

constriction of afferent arterioles by acting on Al receptors [24]. However, systemic hypoxia with limitation of food and water-induced renal alteration has been scarcely investigated. In this study, the results demonstrated that the association of sustained systematic moderate hypoxia with deprivation of food and water caused Scr and BUN elevation, which might be partly due to the dissociation of energy storage, remarkable renal vasodilation in glomerular capillary, interstitial capillary and small vessel with aggregating of red blood cells was observed. In addition, renal tubular cell damage occurred. Whether tubular cell damage is secondary to vascular system changes need further study. In this experiment, similar hemodynamic alterations in other organs such as lungs, liver (will be reported in other article) were also found, indicating that renal hemodynamic changes were part of systemic changes under HDFW.

For the first time, renal pathologic changes in rhabdomyolysis in combination with HDFW were investigated in the present study. Renal tubular damage in animals with associated injury appeared more severe than that with rhabdomyolysis and displayed a different renal damage profile. The time-course and extent of hemodynamic alterations in rhabdomyolysis plus HDFW was similar to that in HDFW alone. Focal necrosis in the cortex occurred only in R/HDFW group. On the basis of these results, it was postulated in the condition of HDFW, renal cells were more vulnerable to rhabdomyolysis-induced myoglobin or heme overload, which might be a major cause of earlier death in the rats with combined injury. This result revealed by our study might be helpful in clinical treatment, for example, the use of vascular dilators likely has negative effects on the outcome, the improvement of circulation and oxygen inhalation deserve more attention.

\section{Conclusion}

Rhabdomyolysis in combination with HDFW-induced renal dysfunction and the functional and structural differences between combined injury and singular injury were investigated, thereby providing new insights for the understanding and treatment of rhabdomyolysis-induced AKI in massive earthquake. However, this study only presented renal changes in rats subjected to rhabdomyolysis in combination with HDFW, there is still much work needed in further research. 


\section{Kidney \\ Blood Pressure Research}

Kidney Blood Press Res 2013;37:414-421

\begin{tabular}{l|l}
\hline DOI: $10.1159 / 000350154$ & (c) 2013 S. Karger AG, Basel
\end{tabular}

Published online: October 10, 2013

www.karger.com/kbr

Wang, et al.: Rhabdomyolysis-induced AKI under hypoxia and deprivation of food and water

\section{Acknowledgements}

This work was funded by The Ministry of Public Security of the People's Republic of China after the Wenchuan earthquake in China.

\section{References}

1 Huerta-Alard' in AL, Joseph Varon J, Marik PE: Bench-to-bedside review: Rhabod-myolysis-an overview for clinicians. Crit Care 2005;9:158-169.

2 Malinoski DJ, Slater MS, Mullins RJ: Crush injury and rhabdomyolysis. Crit Care Clin 2004;20:171-192.

-3 Clyne DH, Kant KS, Pesce AJ, Pollak VE : Nephrotoxicity of low molecular weight serum proteins: physicochemical interactions between myoglobin, hemoglobin, bence-jones proteins and tamm-horsfall mucoprotein. Curr Probl Clin Biochem 1979;9:299-308.

-4 Moore KP, Holt SG, Patel RP, Svistunenko DA, Zackert W, Goodier D, Reeder BJ, Clozel M, Anand R, Cooper CE, Morrow JD, Wilson MT, Darley-Usmar V, Roberts LJ $2^{\text {nd: }}$ : A causative role for redox cycling of myoglobin and its inhibition by alkalinization in the pathogenesis and treatment of rhabdomyolysis-induced renal failure. J Biol Chem 1998;273:31731-31737.

-5 Hsu CH, Kurtz TW, Waldinger TP: Cardiac output and renal blood flow in glycerol-induced acute renal failure in the rat. Circ Res 1977;40:178-182.

6 Ayer G, Grandchamp A, Wyler T, Truniger B: Intrarenal hemodynamics in glycerol-induced myohemoglobinuric acute renal failure in the rat. Circ Res 1971;29:128-135.

7 Giannoglou GD, Chatzizisis YS, Misirli G: The syndrome of rhabdomyolysis: pathophysiology and diagnosis. Eur J Intern Med 2007;18:90-100.

8 Wrogemann K, Pena SD: Mitochondrial calcium overload: a general mechanism for cell-necrosis in muscle diseases. Lancet 1976;1:672-674.

9 Salahudeen AK, Wang C, Bigler SA, Dai Z, Tachikawa H: Synergistic renal protection by combining alkalinediuresis with lipid peroxidation inhibitors in rhabdomyolysis: possible interaction between oxidant and non-oxidant mechanisms. Nephrol Dial Transplant 1996;11:635-642.

10 Rosenberger C, Goldfarb M, Shina A, Bachmann S, Frei U, Eckardt KU, Schrader T, Rosen S, Heyman SN: Evidence for sustained renal hypoxia and transient hypoxia adaptation in experimental rhabdomyolysisinduced acute kidney injury. Nephrol Dial Transpl 2008;23:1135-1143.

11 Bagley WH, Yang H, Shah KH: Rhabdomyolysis. Intern Emerg Med 2007;2:210-218.

12 Holt SG, Moore KP: Pathogenesis and treatment of renal dysfunction in rhabdomyolysis. Intensive Care Med 2001;127:803-811.

13 Abassi A, Hoffman A, Better 0: Acute renal failure complicating muscle crush injury. Semin Nephrol 1998;18:558-565.

14 Vanholder R, Sever MS, De Smet M, Erek E, Lameire N: Intervention of the renal disaster relief task force in the 1999 Marmara, Turkey, earthquake. Kidney Int 2001;59:783-791.

$\checkmark 15$ Feng J, Zen P, Liu Y, Luo J, Jiang W, Peng G: Serum enzyme profile characteristics of victims following the Wenchuan earthquake in China. Clin Chem Lab Med 2009;47:590-595.

16 Richards J: Rhabdomyolysis and drugs of abuse. J Emerg Med 2000;19:51-56.

17 Visweswaran P, Guntupalli J: Rhabdomyolysis. Crit Care Clin 1999;15:415-428.

18 Russell TA: Acute renal failure related to rhabdomyolysis: pathophysiology, diagnosis, and collaborative management. Nephrol Nurs J 2000;27:567-577.

$\checkmark 19$ Better OS, Stain JH: Early management of shock and prophylaxis of acute renal failure in traumatic rhabdomyolysis. N Eng J Med 1990;322:825-827.

20 Odeh M: The role of reperfusion-induced injury in the pathogenesis of the crush syndrome. New Eng J Med 1991;324:1417-1422.

21 Neylon M, Marshall JM, Johns EJ: The effects of systemic hypoxia on renal function in the anaesthetized rat. J Physiol 1995;487:497-511.

22 Marshall JM: Peripheral chemoreceptors and cardiovascular regulation. Physiol Rev 1994;74:543-594.

23 Neylon M, Marshall JM: The role of adenosine in the respiratory and cardiovascular response to systemic hypoxia in the rat. J Physiol 1991;440:529-545.

24 Houck CR, Bing RJ, Craig, FN et al. : Renal hyperemia after the intravenous infusion of adenylic acid, adenosine, or adenosine triphosphate in the dog. Am J Physiol 1948;153:159-175. 